\title{
Evolución en la selección de áreas protegidas en el continente americano: el caso de Estados Unidos, México y Costa Rica
}

\author{
Yesenia Chavarría Olmedo , Ana Laura Martínez García ${ }^{a}$, Elizabeth Ortíz Caballero ${ }^{\mathrm{b}}$, Irene Goyenechea ${ }^{\mathrm{c}, *}$ \\ a Herbario HGOM, Área Académica de Biología, Universidad Autónoma del Estado de Hidalgo/Ciudad del Conocimiento Mineral de la Reforma, Hidalgo, México. \\ ${ }^{b}$ Laboratorio de Paleontología, Área Académica de Biología, Universidad Autónoma del Estado de Hidalgo/Ciudad del Conocimiento Mineral de la Reforma, \\ Hidalgo, México. \\ ${ }^{c}$ Laboratorio de Sistemática Molecular, Área Académica de Biología, Universidad Autónoma del Estado de Hidalgo/Ciudad del Conocimiento Mineral de la Reforma, \\ Hidalgo, México.
}

\begin{abstract}
Resumen
Las áreas naturales protegidas juegan un papel central en la conservación, pero no siempre las propuestas para crear estas áreas se han llevado a cabo usando criterios sistemáticos para su selección y establecimiento, por lo cual en los últimos años se ha evaluado la funcionalidad de estas áreas y se han buscado metodologías que permitan proponer nuevas áreas basadas en el conocimiento integral de la biodiversidad. A continuación se presentan los casos de tres países del continente Americano: Estados Unidos de América, México y Costa Rica, para comparar tres áreas formadas en periodos diferentes así como los criterios que se siguieron para su establecimiento, la evolución de las metodologías implementadas a lo largo del tiempo y el actual éxito de las áreas en la conservación de la biodiversidad. Los tres países cuentan con estrategias de conservación diferentes, basadas en políticas internas y necesidades biológicas, sociales y económicas, por lo que las áreas naturales protegidas en Estados Unidos se enfocan en conservar especies y recursos; en México se busca mantener servicios ecosistémicos y costumbres culturales y en Costa Rica el tema turístico es el más importante a tomar en cuenta para proponer la conservación de áreas.
\end{abstract}

Palabras Clave: Área Natural Protegida, América, criterios, conservación.

\section{Introducción}

La protección de la naturaleza no es un tema reciente, sencillamente ha cobrado un auge proporcional a la fuerza de las acciones que el humano ejerce sobre el ambiente, especialmente durante los últimos decenios (Valle Rodríguez, 2006).

Según Adams (2000), desde hace unos dos mil años en la India se tenían destinadas algunas áreas para la conservación de recursos, mientras que, en Europa, el registro más antiguo data del año 680 D.C., cuando el monje Cuthbert de Lindisfarne declaró a la Isla Inner Farne en el Mar del Norte en la costa inglesa como una "reserva natural" para el pato Eider (Somateria mollissima) (Jones-Walters y Čivić, 2013). En Mesoamérica, los incas, los mayas y los mexicas llevaron a cabo diversas acciones para proteger áreas naturales relevantes. Por ejemplo, la cultura maya vinculó su desarrollo con el bosque tropical, basando sus prácticas agrícolas, hortícolas y forestales en el pluricultivo (Melo, 2002). En México, el rey Nezahualcóyotl, en el siglo XV fundó el primer jardín botánico en Texcoco, por su parte el emperador Moctezuma Xocoyotzin protegió el Bosque de Chapultepec y creó un jardín en El Peñón, los dos en el valle de México; asimismo, estableció jardines en Atlixco Puebla y Oaxtepec Morelos (Melo, 2002).

Pero no fue sino hasta el siglo XIX que se decretó la primera área natural protegida en el mundo: el Parque Nacional de Yellowstone, en 1872 (Culpin, 2003), y a raíz de ese evento se dio paso en todo el mundo a la conservación de áreas con distintos fines, de manera que durante el siglo XX se crearon aproximadamente 30000 áreas protegidas a nivel mundial y actualmente existen más de 120 000, cubriendo una extensión cercana a los $21000000 \mathrm{Km}^{2}$ de tierra y mar; mientras que para América Latina y el Caribe, son casi 2000 las áreas protegidas, reuniendo unos $2400000 \mathrm{Km}^{2}$ en total.

Con el correr del tiempo, se hizo cada vez más evidente que estos lugares estaban llamados a cumplir un papel privilegiado dentro de las estrategias para asegurar la supervivencia humana $-\mathrm{y}$ del resto de las especies- en nuestro entorno planetario. Actualmente se reconoce su papel esencial no sólo como instrumentos para la conservación in situ de la biodiversidad, sino como proveedoras de una vasta gama de servicios ecosistémicos. Así, en diferentes documentos que hoy son considerados verdaderos hitos dentro de la agenda ambiental internacional, las áreas protegidas siempre han estado presentes, de un modo o de otro, como pilares fundamentales para el desarrollo humano sostenible. Por citar sólo algunos, basta con recordar la Estrategia para la Conservación Mundial (1980), Nuestro Futuro Común ("Informe Brundtland", 1987), Cuidar la Tierra (1991), la Estrategia Global para la Biodiversidad (1992) y la Convención de las Naciones Unidas sobre la Diversidad Biológica (1992).

$\mathrm{Si}$ bien la formación de Áreas Naturales Protegidas (ANP's) ya era considerada en las agendas internacionales, fue hasta 1994 cuando la UICN (The World Conservation Union) las define formalmente como "áreas de tierra y/o mar especialmente dedicadas a la protección y mantenimiento de la diversidad biológica, recursos naturales y culturales 
asociados, y manejados a través de medios legales u otros medios efectivos".

Históricamente las primeras reservas se concentraron en paisajes de gran calidad estética y atractivos para el turismo (Mendel y Kirkpatrick, 2002), es decir que fueron seleccionadas sin considerar una metodología específica (Conabio et al., 2007) por lo que en muchos casos dichas áreas no representan el objetivo básico de la conservación. Fue a partir de 1970 que se considera el inicio de la planificación sistemática de la conservación con aportes de diferentes criterios de selección (Koleff et al., 2009). Algunos ejemplos notables de priorización de áreas para la conservación en el ámbito mundial incluyen los hotspots de biodiversidad, las áreas de endemismo de aves (EBA) y los sitios cero extinciones (Ceballos et al., 2009; Koleff et al., 2009).

\subsection{Objetivo}

Examinar el panorama general de los criterios usados en la selección de áreas naturales protegidas en tres momentos de la historia de Estados Unidos, México y Costa Rica para comparar la evolución de dichos criterios de acuerdo a los retos de conservación de cada país.

\section{Estados Unidos}

Estados Unidos es el tercer país más grande del mundo y ocupa el tercer puesto en tamaño poblacional. Alrededor de 200000 especies han sido identificadas en su territorio, lo que representa aproximadamente $13 \%$ del millón y medio de biodiversidad que los taxónomos han reconocido en todo el mundo. A pesar de los esfuerzos realizados en materia de conservación, se calcula que únicamente se conoce el estado de conservación de 21000 especies (cerca de un tercio de la diversidad total), y estas se encuentran en estado vulnerable, en peligro, en peligro crítico o ya extintas (Holsinger, 2001).

Actualmente este país cuenta con 34075 áreas protegidas, de las cuales más del $40 \%$ son manejadas por ministerios subnacionales o agencias. El 12.99\% (1 $233169.299 \mathrm{~km}^{2}$ ) de superficie terrestre total del país forma parte de áreas naturales protegidas, mientras que $3527444.314 \mathrm{~km}^{2}$ (41.06\%), de áreas marinas están protegidas. En este país las ANP's se clasifican en cuatro categorías, según el manejo de tierra en: 1) Áreas de biodiversidad, que tienen protección permanente de la diversidad biológica, no permiten el cambio de uso de suelo, y procuran el mantenimiento de los procesos naturales. En esta categoría se encuentran las áreas naturales para investigación, así como los parques y monumentos nacionales; 2) Áreas naturales, que tienen protección de la cobertura de tierra natural pero permiten algunos usos de los valores naturales, que pueden degradar la biodiversidad. En este grupo se hallan los refugios nacionales de vida salvaje, las áreas silvestres, las áreas de preocupación ambiental crítica y los parques estatales; 3) Áreas de uso múltiple, que tienen poca protección para el cambio de uso de suelo, sujetas a usos extractivos y constantemente ofrecen protección a especies en peligro. Se agrupan aquí las áreas públicas sin designación del Servicio Forestal de los Estados Unidos y de la Oficina de Gestión de Tierras. Finalmente, 4) Áreas de uso intensivo, que tienen poca o ninguna protección legal para el cambio de uso de suelo y que se destinan principal o exclusivamente para el uso humano intensivo. Dentro de esta categoría se encuentra la mayoría de las tierras privadas, las tierras del Departamento de Defensa y las tierras estatales sin designación (Groves, et al., 2000).

\subsection{Parque Nacional de Yellowstone}

Estados Unidos sentó precedente en la historia de la conservación de áreas naturales, cuando proclamó la primera área natural protegida a nivel mundial, el Parque Nacional de Yellowstone.

Este parque ocupa parte de los estados de Wyoming, Idaho y Montana (Figura 1, número 1); inicialmente el área asignada para este propósito fue de 1221773 acres (4 $\left.944.33 \mathrm{~km}^{2}\right)$. La declaración se realizó el 1 de marzo de 1872 como "un área para el disfrute y beneficio de la gente", estableciendo que "toda la madera, depósitos minerales, curiosidades naturales o maravillas que se encuentran dentro deben ser conservadas en su condición natural" (Culpin, 2003). La motivación principal por la que se creó este parque fue el paisajismo y la recreación, sin tomar en cuenta la biodiversidad contenida en él y con la visión de mantener el paisaje exactamente igual, sin incorporar la idea del desarrollo sostenible. Cien años después de su creación, el Parque Nacional de Yellowstone se convirtió en una de las primeras reservas de la biósfera, con una extensión total de $8983 \mathrm{~km}^{2}$. Entre otras cualidades, su importancia reside en sus características geológicas como los géiseres, ya que contiene entre 200 a 250 activos, lo cual representa una cantidad mayor a la que se pueden encontrar en otras partes del mundo. Además, sirve como santuario para más de 1700 especies de plantas, así como 16 de peces, 4 de anfibios, 6 de reptiles, alrededor de 322 especies de aves y por lo menos 61 de mamíferos, algunas de ellas consideradas especies bandera como el bisonte (Bison bison), el oso grizzly (Ursus arctos), el venado cola blanca (Odocoileus virginianus) y el lobo gris (Canis lupus) (Gaston et al., 2008).

\subsection{Parque Nacional de los Everglades}

Con el paso del tiempo, la visión acerca de la biodiversidad y los recursos naturales cambió, de manera que para designar áreas para conservación se comenzó a considerar como prioridad aquellas que presentaran una alta diversidad biológica. Un ejemplo de esto fue la creación del Parque Nacional de los Everglades, una reserva de humedales al sur de la Florida, fundada el 6 de diciembre de 1947 (Figura 1, número 2).

La creación de este parque fue impulsada por Ernest F. Coe, un arquitecto paisajista que, desde 1928 quedó impactado por la singularidad del sitio, y que junto a otros conservacionistas y científicos, fundó la Asociación del Parque Nacional de los Everglades. Estos profesionales contribuyeron al estudio y propuesta de esta área para la conservación, que cuenta con por lo menos seis tipos de hábitat (entre los que destacan los manglares, los pantanos y los bosques de pinos), que sirven como santuario de por lo menos 20 especies raras y en peligro de extinción como: la tortuga laúd (Dermochelys coriacea), el manatí (Trichechus manatus), la pantera de Florida (Puma concolor), el caimán (Alligator mississippiensis) y el gavilán caracolero (Rostrhamus sociabilis), entre otros.

El parque cuenta con una superficie total de $3800 \mathrm{~km}^{2}$, conservando alrededor del $20 \%$ de la extensión original del humedal y es considerado Patrimonio de la Humanidad y 
Reserva Internacional de la Biósfera (National Park Service, 2015).

\subsection{Monumento Nacional Marino de Papahānaumokuākea}

Finalmente, manteniendo la vanguardia en cuanto a conservación en el 2006 se creó el Monumento Nacional Marino de las Islas Noroccidentales de Hawaii, con una superficie de $362000 \mathrm{~km}^{2}$ (Figura 1, número 3). El área se destinó para la protección de las aproximadamente 7000 especies que ahí habitan, de las cuales una cuarta parte son endémicas del lugar.

Con la creación de esta área se protegen especies que se encuentran en peligro crítico de extinción como la tortuga verde (Chelonia mydas), la foca monje hawaiana (Monachus schauinslandi), y el pato Anas laysanensis.

En el año 2016 este monumento fue ampliado a más de un millón y medio de kilómetros cuadrados y cambió su nombre a Monumento Marino de Papahānaumokuākea, convirtiéndose en el ANP más extensa de los Estados Unidos y una de las más grandes a nivel mundial, implementando, además de su objetivo de conservación, programas de investigación científica, marítima y cultural, así como programas educativos y de divulgación (National Ocean Service, 2019).

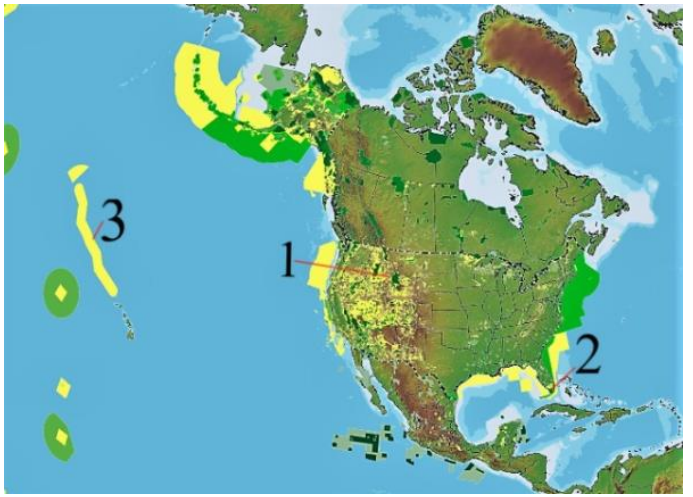

Figura 1: Mapa de las ANP's de Estados Unidos mencionadas. 1) Parque Nacional de Yellowstone, 2) Parque Nacional de los Everglades, y 3 ) Monumento Nacional Marino de Papahānaumokuākea.

\section{México}

En México, a partir del 2002 — con el objetivo de lograr una mayor efectividad en la conservación y una mayor cobertura institucional - la CONANP diseñó un proceso de regionalización de las áreas protegidas que dividió al país en nueve regiones para su atención y siete categorías de ANP's: parques nacionales, reservas de la biósfera, áreas de protección de flora y fauna, santuarios, áreas de protección de recursos naturales monumentos nacionales y humedales (CONANP, 2018). En la actualidad existen 182 ANP's que abarcan una superficie de 90.6 millones de hectáreas, las cuales cubren el $10.78 \%$ de la superficie terrestre y el $22.05 \%$ de la superficie marina del país Fig. 2 (CONANP 2018).

\subsection{Parque Nacional Desierto de los Leones}

El antecedente oficial relacionado con las ANP's en México data de 1876 cuando el presidente Lerdo de Tejada dispuso expropiar, por causa de utilidad pública, la zona boscosa del Desierto de los Leones en la Ciudad de México, declarándose Reserva Nacional Forestal, a fin de proteger los 14 manantiales que desde entonces suministraban agua a la Ciudad de México. Esta misma zona fue decretada en 1917 como el primer parque nacional de México, al considerar de forma adicional la belleza de sus paisajes (Vargas Márquez, 1997).

En 1898, durante el periodo presidencial de Porfirio Díaz, se declaró Bosque Nacional a la región Monte Vedado de Mineral del Chico, Hidalgo, por lo cual dicha área siempre se ha considerado como el primer parque nacional mexicano, aunque tal designación le fue oficialmente otorgada hasta 1982 (Melo, 1997).

La selección de áreas para la creación de parques nacionales, como figura de conservación dominante en ese tiempo, se hizo a partir de tres criterios principales: gran atractivo paisajístico, constituir un potencial recreativo y poseer importancia ambiental para las ciudades próximas (Castañeda-Rincón, 2006).

\subsection{Reserva de la Biósfera Mariposa Monarca}

Entre el periodo de 1970 a 1990 las ANP's se decretaron siguiendo los criterios de ser áreas biogeográficas en las que habiten especies representativas de la biodiversidad nacional, determinando la superficie mejor conservada y denominada como zona núcleo por alojar ecosistemas, fenómenos naturales de importancia especial o especies de flora y fauna que requieran protección (Yañez- Mondragón, 2007) Es en esta etapa cuando se incorporan criterios ecológicosambientales a los planes y programas de gobierno, por lo que la conservación de los recursos naturales empezó a asumirse bajo la perspectiva de un desarrollo sostenible y con mayor rigor jurídico, institucional y apoyo financiero.

Bajo estos lineamientos se decretó, en 1986, la reserva de la Mariposa Monarca como ANP para los fines de migración, hibernación y reproducción de la mariposa monarca, por medio de la combinación de tareas de restauración ecológica, protección, investigación, desarrollo sustentable, difusión, educación ambiental, capacitación y recreación que permitan garantizar la conservación de los bosques templados, así como las condiciones ambientales necesarias para asegurar su ciclo migratorio (CONANP, 2018).

\subsection{Reserva de la Biósfera Barranca de Metztitlán}

A partir del año 2000 y luego del surgimiento de los sistemas de reservas de la biósfera, se da un acercamiento utilitario a la protección de la naturaleza tratando de minimizar el impacto humano sobre el medio ambiente. Este sistema sustituyó al de Parques Nacionales en forma ventajosa, ya que incluye la protección de especies florísticas y animales representativas que los Parques Nacionales no contemplaron como parte de sus funciones de investigación, educación y recreación (Castañeda-Rincón, 2006)

Ejemplo de este enfoque es la Reserva de la Biosfera Barranca de Metztitlán, la cual cuenta con una extensión de 96042.94 hectáreas y fue decretada como ANP el 27 de noviembre del año 2000. En esta área se consideraron características como la representatividad biogeográfica de 
varios ecosistemas terrestres (matorral xerófilo; matorral submontano, bosque de coníferas y bosque tropical caducifolio), por el buen grado de conservación de su biodiversidad,por tener un alto nivel de endemismos, así como la presencia de ecosistemas acuáticos, todo esto aunado a la gran riqueza cultural de la zona, en la que existen construcciones coloniales del siglo XVI, pinturas rupestres, así como gran diversidad gastronómica y artesanal (CONANP, 2003).

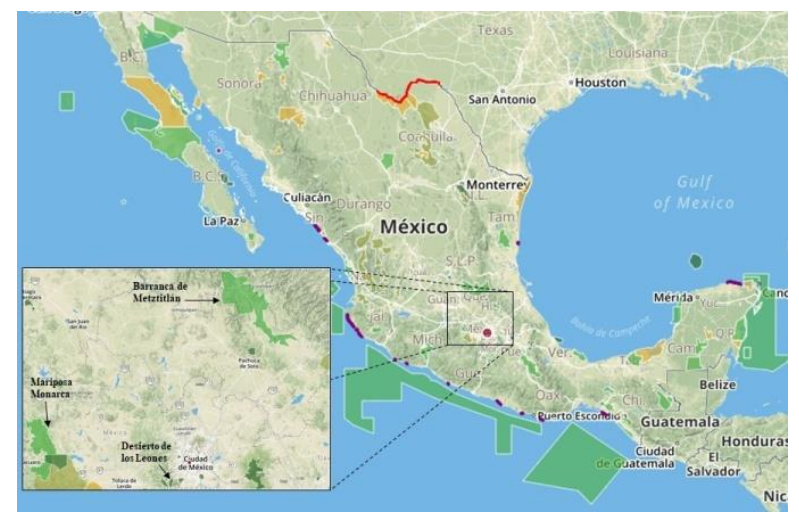

Figura 2: Áreas naturales protegidas de México. En el recuadro se resaltan el Parque Nacional Desierto de los Leones, La Reserva de Biósfera Mariposa Monarca y la Reserva de la Biósfera Barranca de Metztitlán. Tomado y modificado de CONANP 2018.

\section{Costa Rica}

Es un país destacado por su riqueza natural y la creación de un buen sistema de áreas naturales protegidas, con lo que ha obtenido reconocimiento a nivel mundial y convirtiéndolo en modelo de esfuerzos de conservación para otros países (Vargas-Ulate, 2009).

Actualmente el país cuenta con el 26.28\% (1 340872 hectáreas) de superficie continental nacional y el $17.19 \%$ de la superficie marina nacional como área protegida (SINAC, 2010). Territorialmente Costa Rica se divide en 11 áreas de conservación, donde se interrelacionan actividades públicas y estatales (Figura 3). Dentro de estas áreas de conservación se establecen ocho categorías de manejo: los parques nacionales, las reservas biológicas, los monumentos nacionales, los refugios de vida silvestre, las zonas protectoras, las reservas forestales, los humedales y los monumentos naturales.

En este país surgió primero la preocupación por proteger los servicios ecosistémicos como el agua, además de regular la extracción maderera creando así un sin número de leyes y decretos (Porras, 1980; Porras, 1981 y Vargas, 1993). Antes de 1940, el concepto de espacio natural protegido como categoría de manejo no existía en la legislación costarricense (González, 1981). Es a partir, de la participación de Costa Rica en la Convención de Washington de 1942, sobre la "Protección de la flora, fauna y bellezas escénicas de los países de América" que se inició el uso del concepto de espacio natural protegido y se crearon por primera vez en el país, las categorías de manejo de parque nacional, reserva nacional, monumentos naturales y reservas de espacios vírgenes (González, 1981).

Dieciséis años más tarde, en 1958, por la ley número 3763 se ratificó dicha convención y se establecieron espacios protegidos como los parques nacionales volcán Poás, Turrialba e Irazú, cuya administración se le encargó al Instituto Costarricense de Turismo, pero es hasta 1963 que se establece la primera área protegida del país, la Reserva Natural Absoluta Cabo Blanco (Vargas, 2009).

\subsection{Reserva Nacional Absoluta Cabo Blanco}

Creada en 1963, antes del establecimiento del sistema de parques nacionales de Costa Rica (1970), por el sueco Olof Wessberg y su esposa, la danesa Karen Morgenssen, con el propósito de conservar los últimos bosques naturales de la región, que estaban en peligro de desaparecer por la tremenda deforestación en la Península de Nicoya. Esta pareja logró conseguir el interés de organismos internacionales y de instituciones nacionales para financiar el primer proyecto significativo de restauración de tierras deforestadas en Costa Rica (SINAC, 2010).

La fundación de la Reserva de Cabo Blanco marca el momento en que Costa Rica inicia su política de conservación del entorno natural, ya que es la primera área protegida establecida con ese propósito específico. Su creación también fue un hito que impulsó el establecimiento del actual Sistema Nacional de Áreas de Conservación. Actualmente la reserva cuenta con cuatro programas para su administración, en las que se incluyen: investigación, mantenimiento, protección y educación ambiental.

\subsection{Parque Nacional Isla de Coco.}

Creada en 1978, es una de las 160 Áreas Silvestres Protegidas (ASP) que integran el Sistema Nacional de Áreas Protegidas de Costa Rica. El PNIC es mundialmente reconocido como un importante reservorio de biodiversidad y endemismo, sitio de excepcional belleza paisajística y uno de los mejores destinos para el buceo recreativo.

El parque también cuenta con sus propios programas: protección, administración, investigación-monitoreo y uso público (Plan de Monitoreo del Impacto del Turismo Marino en el Parque Nacional Isla del Coco, 2016).

\subsection{Parque Nacional los Quetzales}

Es un área de conservación natural, cuya creación fue el 16 de enero del 2006. Los Quetzales fue creado para conservar los ambientes naturales de altura (en especial de los robledales, los bosques nublados y el páramo), la función hidrológica de estos bosques y proteger de los hábitats de especies endémicas y de reproducción del quetzal. La consolidación de este Parque pretende llevar una salida económica, de la mano de la conservación, a las comunidades aledañas, entre ellas las de Providencia y San Gerardo de Dota. 


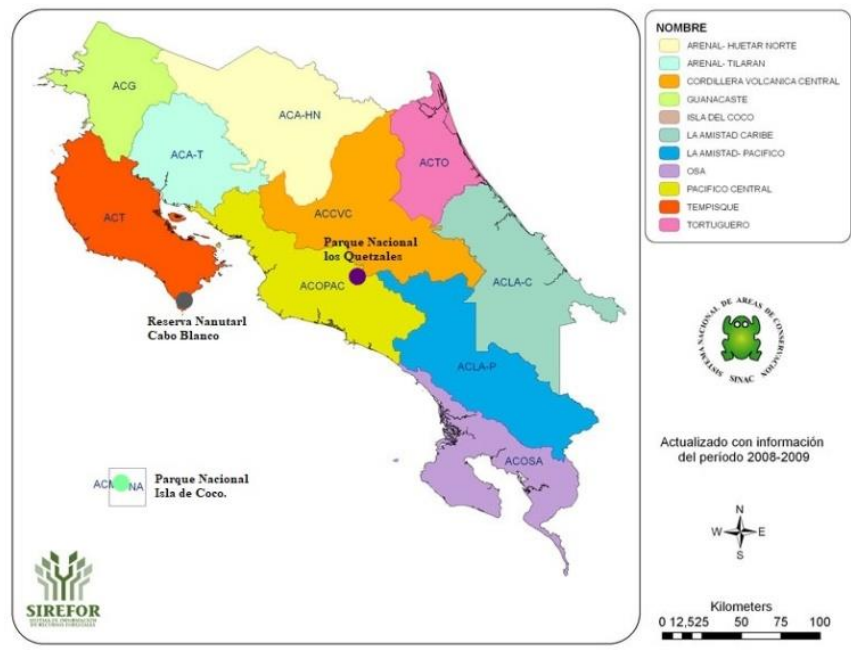

Figura 3: Mapa de Costa Rica con la división de sus 11 áreas conservación y señalando con puntos la ubicación de las ANP's, en morado el Parque Nacional los Quetzales, en Gris la Reserva Nacional Absoluta Cabo Blanco y en verde el Parque Nacional Isla de Cocos. Tomado y modificado de el Sistema de Información de los Recursos Forestales de Costa Rica (SERIFOR)

\section{Comparación entre países y evolución de las metodologías.}

Los tres países cuentan con estrategias de conservación diferentes, basadas en políticas internas y necesidades biológicas, sociales y económicas. Cada país cuenta con categorías de ANP que, aunque en el nombre pueden ser equivalentes, en su objetivo y misión no lo son. Entre México y Costa Rica existen más similitudes, ya que ambos tienen planes de manejo para cada una de sus áreas, mientras que, en Estados Unidos clasifican las ANP's de acuerdo al uso de suelo permitido.

Estos tres países tienen en común que, en un inicio la selección de áreas fue hecha bajo el mismo principio, una metodología cualitativa en la cual los principales criterios para la selección fueron el valor escénico, los usos recreativos, la presencia o ausencia de aprovechamiento forestal o la disposición del terreno (Ceballos et al. 2009; Conabio et al.2007; Koleff y Urquiza 2011; Margules el al.2002). Este tipo de metodologías tenían la ventaja de ser simples, rápidas y económicas. Yellowstone, El desierto de los Leones y Cabo Blanco en su momento cumplieron con el propósito para el que fueron creadas, pero con el tiempo se hizo evidente que presentaban una visión limitada, estrecha y a largo plazo, inoperante para la conservación (Toledo, 2005).

Con la evaluación y detección de posibles fallas en el funcionamiento de las ANPs fue posible replantear la efectividad y los objetivos con que fueron originalmente propuestas (Castaño-Villa, 2005), lo anterior permitió en el caso de Yellowstone, por ejemplo, modificar su categoría de Parque Nacional a Reserva Internacional de la Biósfera en el año de 1976, así como la incorporación de programas de investigación, mantenimiento, protección y educación ambiental en Cabo Blanco.

$\mathrm{Al}$ incorporar criterios ecológicos-ambientales a los planes y programas de gobierno, surgió una nueva etapa, donde la conservación de los recursos naturales fue más eficiente ya que se enfocó en áreas biogeográficas donde habitan especies representativas de la biodiversidad, fue durante este periodo cuando se decretaron el Parque Nacional de los Everglades, La Reserva de la Biósfera Mariposa Monarca y el Parque Nacional Isla de Cocos, con el fin de conservar la diversidad biológica y así servir como santuarios de las especies de flora y fauna además de fenómenos naturales de importancia especial que requerían protección, como es la migración de la mariposa monarca o la protección de las especies endémicas de los Everglades y la Isla de Cocos.

Posteriormente, comenzó a asumirse la perspectiva de un desarrollo sostenible; este enfoque incluye además del objetivo de la conservación de flora y fauna, programas de investigación científica, educativos, de divulgación y de recreación. Bajo este enfoque fueron creados a partir del año el Monumento Nacional Marino de (Papahānaumokuākea, 2000), la Reserva de la Biósfera Barranca de Metztitlán y el Parque Nacional los Quetzales, logrando una mayor eficiencia al permitir la interacción de diferentes sectores de la sociedad. Este enfoque permitió en el año 2016 la ampliación del Monumento Nacional Marino de Papahānaumokuākea, además de incorporar programas de investigación científica, marítima, cultural, así como programas educativos y de divulgación.

En la actualidad ante la creciente crisis ambiental se ha buscado el uso de metodologías cuantitativas más exactas (Chávez-González et al. 2014) y con el transcurso de los años se ha observado una notable evolución en las propuestas de ANP's. Los primeros pasos se dieron cuando comienzan a aparecer áreas prioritarias para la conservación utilizando metodologías como:

1. Subrogados de la biodiversidad, principalmente enfocados a especies (por ejemplo: especies amenazadas, endémicas, raras o en peligro).

2. Criterios ecológicos: como la diversidad, rareza o singularidad, integridad, representatividad, fragilidad o vulnerabilidad, conectividad $\mathrm{y}$ dimensiones.

Lo cual ha permitido comparar, homologar rangos y valorar las ANP's (Mallarach, 2000).

Como en la ciencia el conocimiento nunca es estático ni absoluto, estas metodologías también han sido puestas a prueba, muchas de ellas mostrando ventajas para ciertos grupos y otras han mostrado ineficiencia, surgiendo así nuevas corrientes en el tema de la conservación. Dichas áreas, por mencionar solo algunas, son la filogenética y la biogeografía, que si bien no son áreas de reciente surgimiento, sus metodologías han sido empleadas para la conservación y hasta el momento han mostrado gran eficiencia al combinarse con herramientas estadísticas y tecnológicas de última generación.

\section{Conclusión}

A pesar de la evolución a lo largo de los años de las metodologías y de los múltiples criterios propuestos para la elección de áreas, poco han sido empleadas al momento de decretar áreas, en su mayoría solo han sido empleadas metodologías basadas en la riqueza de especies o por subrogados. $\mathrm{Y}$ aunque en la actualidad existen distintas disciplinas (índices estadísticos, diversidad genética, 
filogeografía, filogenia, biogeografía, modelos de cambio climático, entre otros) que podrían ayudar a la elección de ANP's de una manera eficiente y óptima estas no han sido aplicadas, debido a la nula comunicación entre los generadores del conocimiento y los tomadores de decisiones.

En la actualidad cada país cuenta con categorías de ANP's diferentes, elegidas por los atributos propios de cada zona y las situaciones socio-políticas particulares de cada nación, por lo cual su eficiencia es muy variable y difícil de estandarizar (Bruner, 2001). Estados Unidos se enfoca en conservar especies y recursos, México busca mantener servicios ecosistémicos y costumbres culturales, mientras que Costa Rica toma muy en cuenta el tema turístico en la conservación de áreas

México ha enfrentado el compromiso de la conservación, ampliando los análisis con varios enfoques, a escalas más detalladas y sumando esfuerzos con varias instituciones. Los pocos estudios disponibles para determinar las áreas requeridas para mantener los procesos ecológicos o las poblaciones viables de especies nativas, indican que la superficie dedicada a la conservación debería incrementarse de dos a seis veces (Conabio et al., 2007). Esto, hace pensar en las dificultades que aún enfrentan los gobiernos, si bien es fundamental fortalecer las redes de áreas naturales protegidas federales, estatales, municipales y las reservas privadas, se requiere de un conjunto diversificado y complementario de otros instrumentos de conservación para asegurar la permanencia y funcionamiento adecuado de los ecosistemas.

\section{English Summary}

Evolution in the selection of Protected Areas within the American Continent: study cases of the United States, Mexico and Costa Rica

\footnotetext{
Abstract

Natural protected areas play a key role in conservation, but the proposals to create these areas have not always been conducted using systematic criteria for their selection and establishment. Recently, the functionality of these areas have been evaluated, and methodologies that allow the proposal of new areas based on the comprehensive understanding of biodiversity have been recommended. Here we show the cases of three American countries: United States of America, Mexico and Costa Rica, to compare three areas conformed in different periods, as well as the criteria followed for its establishment, the evolution of the methodologies implemented over time and the current success of the areas in the conservation of biodiversity. The three countries have different conservation strategies, based on internal policies and biological, economic, and social needs so the protected natural areas in the United States focus on preserving species and resources; Mexico seeks to maintain ecosystemic services and cultural customs while and in Costa Rica tourism is the most important issue to take into account when proposing conservation areas.
}

Keywords:

Natural protected area, America, criteria, conservation.

\section{Agradecimientos}

Este trabajo se realizó como parte del curso de posgrado "Métodos biogeográficos para la conservación". YCO, AMG y EOC agradecen el apoyo de CONACYT por la beca para realizar los estudios de posgrado.

\section{Referencias}

Adams, W. M. 2000. The green web: A union for world conservation. Journal of Environmental Policy and Planning, 2(3):261-262.

Bruner, A. G., E. R. Gullison., E. E. Rice, \& G. A. B. Da Fonseca., 2001. Effectivenees of Parks in Protecting Tropical Biodiversity. Science. Vol. 291. 125-127 p.

Rincón, J. C. 2006. Las áreas naturales protegidas de México; de su origen precoz a su consolidación tardía. Scripta Nova: revista electrónica de geografía y ciencias sociales, 10 .

Castaño-Villa, G. J. 2006. Áreas protegidas, criterios para su selección y problemáticas en su conservación. Boletín Científico - Centro de Museos -Museo de Historia Natural 10: 79-101.

Ceballos, G., E. Díaz P., H. Espinosa, O. Flores V., A. García, L. Martínez, E. Martínez M., A. Navarro, L. Ochoa, I. Salazar y G. Santos B. 2009. Zonas críticas y de alto riesgo para la conservación de la biodiversidad de México. En: Sarukhán, J. (coord.). Capital natural de México vol. II: Estado de conservación y tendencias de cambio. Conabio, México, D.F., México. 575-600 p.

Chávez-González, H., M. J. González-Guillén y P. Hernández de la Rosa. 2014. Metodologías para identificar áreas prioritarias para la conservación de ecosistemas naturales. Revista Mexicana de Ciencias Forestales. 6(27): 8-23.

Comisión Nacional para el Conocimiento y Uso de la Biodiversidad (Conabio)- Comisión Nacional de Áreas Naturales Protegidas (Conanp)-The Nature Conservancy (TNC)-Pronatura-Facultad de Ciencias Forestales, Universidad Autónoma de Nuevo León (FCFUANL). 2007. Análisis de vacíos y omisiones en conservación de la biodiversidad terrestre de México: Espacios y especies. Talleres Gráficos de México, México, D.F., México. 128 p

CONANP. 2003. Programa de Manejo. Reserva de la Biósfera Barranca de Metztitlán. Comisión Nacional de Áreas Naturales Protegidas. México. 198 p.

CONANP. 2018. Áreas Naturales Protegidas. Región Centro y Eje Neovolcánico.[Octubre 2018] https://www.gob.mx/conanp

Culpin, M. S. 2003. "For the Benefit and Enjoyment of the People": A History of the Concession Development in Yellowstone National Park, 1872-1966. National Park Service, Yellowstone Center for Resources, Yellowstone National Park, Wyoming, YCR-CR-200301.

Gaston, K. J., Jackson, S. F., Cantú-Salazar, L. y G. Cruz-Piñón. 2008. The Ecological Performance of Protected Areas. Annual Review of Ecology Evolution and Systematics 39:93-113.

González, R. 1981. "Contribution a l'étude du droit de la protection de la nature et del'environnement au Costa Rica". [Tesis de doctorado en Derecho]. Francia: Université de Bordeaux II.

Groves, C. R., Kutner, L. S., Stoms, D. M., Murray, M. P., Scott, J. M., Schafale, M., Weakley, A. S. y R. L. Pressey. 2000. Owning up to our responsibilities, Who owns lands important for biodiversity? En: Stein, B. A., Kutner, L. S. y J. S. Adams (eds.) Precious Heritage The Status of Biodiversity in the United States. Oxford University Press, Nueva York, 399 p.

Holsinger, K. E., 2001. Biodiversity in the United States. BioScience, 51(3), 256. doi:10.1641/0006-3568(2001)051[0256:bitus]2.0.co;2 
Jones -Walters, L., Čivić, K. 2013. European protected areas: Past, present and future. Journal for Nature Conservation, 21(2): 122-124.

Koleff, P., M. Tambutti, I. J. March, R. Esquivel, C. Cantú y A. Lira N. 2009. Identificación de prioridades y análisis de vacíos y omisiones en la conservación de la biodiversidad de México. In: Sarukhán, J. (coord.). Capital natural de México vol. II: Estado de conservación y tendencias de cambio. Conabio. México, D.F., México. 651-718 p.

Koleff, P. y T. Urquiza H. (coords.). 2011. Planeación para la conservación de la biodiversidad terrestre en México: Retos en un país megadiverso. Comisión Nacional para el Conocimiento y Uso de la Biodiversidad-Comisión Nacional de Áreas Naturales Protegidas. México, D.F., México. 250 p.

Mallalarch, J. M., 2000. Evaluación de la gestión de los espacios naturales protegidos: Criterios, métodos y retos. En: J. Gómez-Limón García y M. Múgica de la Guerra (Editores). Actas del $5^{\circ}$ Congreso de EUROPARC-España. ESPARC' 99. 10-20 p.

Margules, C. R., R. L. Pressey and P. H. Williams. 2002. Representing biodiversity: data and procedures for identifying priority areas for conservation. Journal of Bioscience 27 (4):309-326.

Melo, G., 1977. Desarrollo de los parques nacionales mexicanos. Tres estudios sobre el mismo tema. Serie Varia. Vol. I núm. 3. Instituto de geografía. UNAM. México. Pp. 58-154.

Melo, C. 2002. Áreas Naturales Protegidas de México en el Siglo XX México: Instituto de Geografía, Universidad Nacional Autónoma de México, Temas selectos de Geografía de México, 1. Textos monográficos: 6. Medio ambiente. $156 \mathrm{p}$.

Mendel, L. C. y J. B. Kirkpatrick. 2002. Historical progress of biodiversity conservation in the protected-Area system of Tasmania, Australia. Conservation Biology, 16(6): 1520-1529.

National Ocean Service. 2019. Papahānaumokuākea Marine National Monument.

Recuperado https://www.papahanaumokuakea.gov/about/

National Park Service. 2015. Everglades National Park Florida History \& Culture. Recuperado https://www.nps.gov/ever/learn/historyculture/index.htm
Porras, A. 1980. "Análisis general de la legislación forestal". Revista de Ciencias Jurídicas 14: 164 -173.

Porras, A. 1981. "Derecho ambiental de Costa Rica". Revista Judicial 20: 83-86.

SINAC (Sistema Nacional de Áreas de Conservación, CR). 2018. Guía Rápida para la Implementación de la Zonificación en Áreas Silvestres Protegidas de Costa Rica. Ed. Artavia G, San José, Costa Rica. 28 p.

Sistema Nacional de Áreas de Conservación-SINAC del Ministerio de Ambiente, Energía y Telecomunicaciones-MINAET 2010. Políticas para las Áreas Silvestres Protegidas (ASP) del Sistema Nacional de Áreas de Conservación-SINAC 2011-2015. San José CR 44 p.

Toledo, V. M. 2005. Repensar la conservación:¿ áreas naturales protegidas o estrategia bioregional?. Gaceta ecológica, (77), 67-83.

Valle-Rodríguez, S. 2006. Las Áreas Naturales Protegidas en México. Un ejemplo de propuesta de gestión de un área protegida y plan de manejo en "La Sierra" de Monte Escobedo (Zacatecas, México). Tesis Doctoral. Instituto de Ciencia y Tecnología Ambiental. Universidad Autónoma de Barcelona.

Vargas, G. 1993. "La protección de los recursos naturales en un país subdesarrollado: caso de Costa Rica". Revista de Ciencias Sociales 59: 81-94.

Vargas-Márquez, F. 1997. Parques Nacionales de México: Aspectos físicos, sociales, legales, administrativos, recreativos, biológicos, culturales, situación actual y propuestas en torno a los parques nacionales de México. México, D. F.: Secretaria de Medio Ambiente, Recursos Naturales y Pesca.

Vargas-Ulate, G. 2009. Turismo y espacios naturales protegidos en Costa Rica: enfrentamiento o concentración. Rev. Ciencias Sociales 123: 49-78.

Yáñez-Mondragón, C. F. 2007. Las áreas naturales protegidas en México, criterios para su determinación Caso estudio: Sierra Tarahumara, Estado de Chihuahua. Academia de Ingeniería. México. 